\title{
Technical Note: Real-Time Diagnosis of the Hygroscopic Growth Micro-Dynamics of Nanoparticles with Two-Dimensional Correlation Infrared Spectroscopy
}

\author{
Xiuli Wei ${ }^{1,2}$, Haosheng Dai ${ }^{1,2}$, Huaqiao Gui ${ }^{1,3}$, Jiaoshi Zhang ${ }^{1}$, Yin Cheng ${ }^{1,2}$, Jie
}

\author{
Wang ${ }^{1}$, Yixin Yang ${ }^{1}$, Youwen Sun ${ }^{1}$, and Jianguo Liu ${ }^{1,2,3}$
}

1 Key Laboratory of Environmental Optics and Technology, Anhui Institute of Optics and Fine Mechanics, Hefei Institutes of Physical Science, Chinese Academy of Sciences, Hefei 230031, China

102 University of Science and Technology of China, Hefei 230031, China

3 CAS Center for Excellence in Regional Atmospheric Environment, Institute of Urban Environment, Chinese Academy of Sciences, Xiamen 361021, China

Correspondence to: hqgui@aiofm.ac.cn (Huaqiao Gui) and ywsun@aiofm.ac.cn(Youwen Sun)

\section{Abstract}

Nanoparticles can absorb water to grow up and this will affect the light scattering behavior, cloud condensation nuclei properties, lifetime, and chemical reactivity of these particles. Current techniques usually assume the shapes of nanoparticles to be spherical in calculation of aerosol liquid water content (ALWC), which may result in large uncertainties when the shapes of nanoparticles show large deviations to the spherical assumptions. Furthermore, current techniques are also difficult to identify the intermolecular chemical interactions of phase transition micro-dynamics during nanoparticle deliquescence process because their limited temporal resolutions are unable to capture the complex femtosecond-level intermediate states. In this study, the hygroscopic growth properties of nanoparticles with electrical mobility diameter of approximately $100 \mathrm{~nm}$ and their phase transition interaction dynamics on molecular scale are characterized on real time by using the Fourier transform infrared (FTIR) and the two-dimensional correlation infrared (2D-IR) spectroscopic techniques. With the FTIR spectroscopy, we develop a novel real-time method for ALWC by constructing the absorption spectra of liquid water, and realized real-time measurements of water content and dry nanoparticle mass to characterize the hygroscopic growth factors (GF) which show discrepancies to the extended aerosol inorganics model (E-AIM). We further explore the difference that the deliquescence points of sodium nitrate $(\mathrm{SN})$ and oxalic acid (OA) compounds are lower than that of AS by using the 2D-IR 
the hydration interactions and investigate the dynamic deliquescence process of the functional groups for AS and its mixture compounds. Both SN and OA compounds lower the deliquescence point of AS, but only AN can change the hydrolysis reaction mechanism for AS in AS/AN and AS/OA mixtures. This study can not only provide important information with respect to the difference in phase transition point under different conditions, but also improve current understanding of the chemical interaction mechanism between nanoparticles (particularly for organic particles) and medium, which is of great significance for haze control across China.

Keywords: Nanoparticles; phase transition micro-dynamics; two-dimensional correlation infrared spectroscopy; hydration interactions; functional groups

\section{Introduction}

Nanoparticles have long atmospheric lifetimes of weeks to months. As the increase in relative humidity, the sizes of nanoparticles will grow up due to the absorption of water, which may have complex phases and mixing states(Riemer et al., 2019) that influence the light scattering behavior, cloud condensation nuclei properties, lifetime, and chemical reactivity of the particles(Lee and Allen, 2012; Vogel et al. 2016; Abbott and Cronin, 2021). An improved knowledge of these complex phases and states are crucial for investigating the gas-particle interactions in the atmosphere. Since the particle size vs. water uptake relationship is influenced by mixing characteristics of various inorganic and organic compounds (Nguyen et al., 2016;Steinfeld and Pandis, 2016), characterizing the water-aerosol interactions is also critical for identifying the fate and transport of trace species in the Earth's system and their effects on air quality, radiative forcing, and regional hydrological cycling(Carlton et al., 2020; Fan et al., 2018).

Ammonium sulfate is an important constituent and a major source of atmospheric nanoparticles originated from anthropogenic activities(Ruehl et al., 2016; Kirkby et al., 2011; Xu et al., 2020). Various techniques such as the hygroscopic tandem differential mobility analyzer (H-TDMA), the electrodynamic balance (EDB), and the environmental scanning electron microscope (ESEM) have been used to investigate the 
65 hygroscopicity of ammonium sulfate (Tang and Munkelwitz, 1977; Tang and Munkelwitz, 1994; Gysel et al.,, 2002; Matsumura and Hayashi 2007). These methods can characterize the deliquescence or phase transition processes of particles down to nanoscale. However, they usually assume the shapes of nanoparticles to be spherical in calculation of aerosol liquid water content (ALWC), which may result in large uncertainties when the shapes of nanoparticles show large deviations to the spherical assumptions. Furthermore, current techniques are also difficult to identify the intermolecular chemical interactions of phase transition micro-dynamics during nanoparticle deliquescence process because their limited temporal resolutions are unable to capture the complex femtosecond-level intermediate states.

Recent studies concluded that the phase transition processes of particles may include multiple intermediate states and are more complex than those indicated in previous results. These intermediate states differ from one to the other and last less than $10 \mathrm{~ms}$ (Esat et al., 2018). A label-free photonic microscope which uses Bloch surface waves as its illumination source for imaging and sensing is capable to provide real-time measurements of the hygroscopic growth process of a single aerosol with particle diameter of less than $100 \mathrm{~nm}$ (Kuai et al., 2020). This method can provide valuable insights into the deliquescence and phase transition mechanisms of particles but cannot determine chemical composition information of particle deliquescence or growth or phase transition processes. It is necessary to develop a method to characterize the intermolecular interaction mechanisms during hygroscopic growth of nanoparticles, which is crucial to understand the physicochemical properties of atmospheric aerosol and the nanoparticle-water interactions in hygroscopic growth, and further for haze control purpose.

In this study, the hygroscopic growth properties of mixed nanoparticles containing $\left(\mathrm{NH}_{4}\right)_{2} \mathrm{SO}_{4} / \mathrm{NaNO}_{3}$ (ammonium sulfate (AS)/sodium nitrate (AN)) and $\left(\mathrm{NH}_{4}\right)_{2} \mathrm{SO}_{4} / \mathrm{oxalic}$ acid (AS/OA) and the phase transition interactions of these particles on molecular scale are characterized on real time by using the Fourier transform infrared (FTIR) and the two-dimensional correlation infrared (2D-IR) spectroscopic techniques. We use a FTIR spectrometer and an extended aerosol inorganics model (E- 
AIM) to characterize and predict the hygroscopic growth of pure AS particle and the AS/AN and AS/OA mixed particles, respectively. We further use the 2D-IR spectroscopic technique to analyze the intermolecular interactions of the phase transition with respect to different levels of relative humidity (RH). This study can not only provide important information with respect to the difference in phase transition point under different conditions, but also improve current understanding of the chemical interaction mechanism between nanoparticles (particularly for organic particles) and medium, which is of great significance for haze control across China.

\section{Material and method}

\subsection{Experiment description}

105 The experimental system includes a nanoparticle generation system, a humidification system, and a FTIR analysis system. Nanoparticles with diameters of $\sim 100 \mathrm{~nm}$ (volume equivalent diameter (Dve)) are aerosolized by an atomizer (model 255, MetOne), dried by a diffusion dryer (model 3062, TSI), sorted into specific diameters (Dve) by a differential mobility analyzer (DMA; model 3082, TSI), and finally deposited onto a $3 \mathrm{~cm} \times 3 \mathrm{~cm}$ zinc selenide ( $\mathrm{ZnSe}$ ) substrate (Figure 1) inside a sample cell through a cone-shaped hole. The sheath-to-sample flow ratio of the DMA is set to be $10: 1$ (the sheath flow is $10 \mathrm{~L} / \mathrm{min}$ and the sample flow is $1 \mathrm{~L} / \mathrm{min}$ ), which can produce an effective mobility for the measured aerosol with size ranging from 14.9 to $673.2 \mathrm{~nm}$. The nanoparticles with a $D v e$ of $\sim 100 \mathrm{~nm}$ are selected for deposition. After

115 about a deposition time of $12 \mathrm{~h}$, the substrate is sealed inside the sample cell to obtain a stable humidity condition for subsequent analysis. The humidification system(Kuai et al., 2020) can provide a specific RH for the sample cell with a precision of $0.1 \%$. The FTIR spectrometer (Tensor 27, Bruker Optics, Germany) starts to take absorption spectra of the samples approximately 5 min after the injection of each designated $\mathrm{RH}$. This time interval is used to stabilize the atmospheric condition inside the sample cell.

The FTIR spectrometer is equipped with a $\mathrm{KBr}$ beam splitter and a liquid nitrogencooled mercury cadmium telluride (MCT) detector for measuring the absorption spectra of the samples. A He-Ne laser metrology keeps the FTIR instrument in a good optical alignment. The FTIR spectrometer saves middle infrared (MIR) spectra with a spectral range of 800 to $4000 \mathrm{~cm}^{-1}$, spectral resolution of $4 \mathrm{~cm}^{-1}$, and repeat times of 64 . The infrared spectra are first subjected to a baseline correction with Opus 7.0 software, and 
then are used to derive the liquid water content using the optical constants of water in the infrared provided by Downing and Williams(Downing and Williams, 1975). We quantify the nanoparticles masses using a simple procedure described in our previous studies without any treatment to the substrates (Wei et al., 2019).

After baseline correction, the infrared spectra are normalized into 2D-IR spectra with the 2D Shige software (Kwansei-Gakuin University, Japan)(Noda and Ozaki, 2014). The wavenumber regions ranging from $2800-3800 \mathrm{~cm}^{-1}$ and from 800 $1400 \mathrm{~cm}^{-1}$ which cover the absorption features of almost all identifiable functional groups of interest are selected for analysis. In present work, the red and blue colors in the 2D-IR spectra represent positive and negative correlations, respectively.

\subsection{Sample description}

In this study, all chemical reagents are produced by Aladdin Reagent Inc. (reagent grade, $99.8 \%$ purity), and the water is obtained from an ultrapure water system (DirectQ3, Millipore). Table 1 lists all chemicals used in the experiment and their concentrations. The single components were dissolved individually in ultrapure water with a concentration of $4.0 \mathrm{~g} / \mathrm{L}$. The mixture (including AS/AN and AS/OA) solutions were prepared by mixing the two required single components with a mass ratio of 1:1. AS is selected as the representative inorganic salt and OA is an important water-soluble organic compound contained in atmospheric aerosols. We select AS as the representative inorganic salt because it is a significant component of the submicronscale aerosol mass in the atmosphere. In addition to an important water-soluble organic compound contained in atmospheric aerosols (Wang et al., 2019), OA is also the dominant dicarboxylic acid in both urban and remote atmospheric aerosols (Richards et al., 2020).

\subsection{Methodology}

The hygroscopic growth factor (GF), indicating the water uptake ability of aerosol particles, is defined as GF $=D_{\text {wet }} / D_{0}$, where $D_{\text {wet }}(\mathrm{cm})$ is the mean diameter of the particles at the designated $\mathrm{RH}$ and $D_{0}(\mathrm{~cm})$ is the mean initial diameter of the dry

155 particles at room temperature. In present work, room temperature is assumed to be $25^{\circ} \mathrm{C}$ and the $\mathrm{RH}$ varies from $50 \%$ to $95 \%$. The GF used for investigation of hygroscopic growth properties of nanoparticles can be calculated via equations (1) to (4), 


$$
\begin{aligned}
& V_{\text {water }}=\frac{M_{\text {water }}}{\rho_{\text {water }}} \\
& V_{0}=\sum_{\mathrm{i}}\left(\frac{M_{\mathrm{i}}}{\rho_{\mathrm{i}}}\right)=\sum_{\mathrm{i}}\left(V_{\mathrm{i}}\right) \\
& V_{\text {wet }}=V_{0}+V_{\text {water }} \\
& G F=\frac{D_{\text {wet }}}{D_{0}}=\left(\frac{V_{\text {wet }}}{V_{0}}\right)^{1 / 3}
\end{aligned}
$$

Where $V_{0}\left(\mathrm{~cm}^{3}\right)$ is the initial volume of the dry nanoparticle at approximately $25^{\circ} \mathrm{C}$, and $V_{\text {water }}\left(\mathrm{cm}^{3}\right)$ is the water volume contained in the nanoparticle at the designated $\mathrm{RH}$; $M_{\text {water }}(\mathrm{g})$ and $M_{i}(\mathrm{~g})$ are the calculated water mass and the mass of the $\mathrm{i}^{\text {th }}$ pure component at the designated RH, respectively; $\rho_{\text {water }}\left(\mathrm{g} / \mathrm{cm}^{3}\right)$ (approximately $1 \mathrm{~g} / \mathrm{cm}^{3}$ ) and $\rho_{i}\left(\mathrm{~g} / \mathrm{cm}^{3}\right)$ are the densities of water and the $i^{\text {the }}$ pure component, respectively, and $i$ is the number of pure component.

We use the E-AIM (UNIFAC) following the Zdanovski-Stokes-Robinson (ZSR) method to predict GF (http://www.aim.env.uea.ac.uk/aim/aim.php). The basis of this method is described as,

$$
\begin{aligned}
& G F_{\text {mix }}=\sum_{\mathrm{i}}\left(\varepsilon_{\mathrm{i}} G F_{\mathrm{i}}^{3}\right)^{1 / 3} \\
& \varepsilon_{\mathrm{i}}=\frac{V_{\mathrm{i}}}{\sum_{\mathrm{i}} V_{\mathrm{i}}}
\end{aligned}
$$

Where $\varepsilon_{\mathrm{i}}$ is the volume fraction of the $i^{\text {th }}$ pure component in the dry mixture, $G F_{i}$ is the GF of the $i^{\text {th }}$ pure component.

\section{3. Results and discussion}

\subsection{Spectral characteristics of nanoparticles during hygroscopic growth process.}

Figure 2 shows the FTIR spectral characteristics of the AS nanoparticles under humidity conditions from $45 \%$ to $92 \%$. Figure 3 compares the predicted $M_{\text {water }} / M_{0}$ ( $M_{\text {water }}$ is the mass of liquid water in the nanoparticles; $M_{0}$ is the initial mass of nanoparticles) ratio after deliquescence from E-AIM (UNIFAC model) (http://www.aim.env.uea.ac.uk/aim/aim.php) and the measured hygroscopic growth properties from the FTIR spectra for the AS particles. The results show that the 
predicted and measured $M_{\text {water }} / M_{0}$ results are generally consistent throughout the humidification process. The strong peaks observed at $3250 \mathrm{~cm}^{-1}$ and $1112 \mathrm{~cm}^{-1}$ at the initial $\mathrm{RH}$ of $45 \%$ are the stretching vibration peak of $\mathrm{OH}$ and the symmetrical stretching vibration $\left(v_{s}\right)$ peak of the sulfate, respectively(Wang et al., 2017; Nájera and Horn, 2009; Gopalakrishnan et al., 2005). With the increase in RH between $45 \%$ and $80 \%$, the peak position of the symmetrical stretching vibration of the sulfate $\left(1112 \mathrm{~cm}^{-1}\right)$ starts to redshift slowly (Figure 3), which indicates that water molecules have been attached to the surface of the solid AS, and the sulfate is then bonded with these water molecules to form a hydrogen bond during this hydration process(Yeşilbaş and Boily, 2016). The area of $\mathrm{OH}$ reflects the liquid water content in the $\mathrm{ZnSe}$ substrate. We find that, in the meantime, the area and position of the $\mathrm{OH}$ stretching peak did not change significantly, which indicates that no hygroscopic growth of the AS nanoparticles occurs. All these behaviors are captured on real time by the FTIR spectra. (Wang et al., 2019; Tang et al., 2016; Nájera and Horn 2009; Martin 2000)

When the RH reaches $80 \%$, the peak position of the sulfate shifts to $1099 \mathrm{~cm}^{-1}$, the $\mathrm{O}-\mathrm{H}$ stretching peak is still the same as that in initial humidity condition $(45 \%)$ but the area of the peak increase abruptly from 0.25 to 5.47 (Figure 3). This indicates that the nanoparticles have absorbed water rapidly and transformed from the crystalline phase to the aqueous phase. According to the E-AIM predictions and the results from previous studies (Estillore et al. , 2016; Cruz and Pandis, 2000; Tang 1982), this process is called the deliquescence, and the $\mathrm{RH}$ at this stage is referred to as the deliquescence $\mathrm{RH}$ (DRH).

205 When deliquescence occurs, $\mathrm{NH}_{4}{ }^{+}$molecules hydrated with $\mathrm{SO}_{4}{ }^{2-}$ are replaced with $\mathrm{H}_{2} \mathrm{O}$ molecules, which leads to the redshift in the symmetrical stretching vibration peak for the sulfate (Dong et al., 2007). Tang et al. (1982), Cruz and Pandis (2000), and Estillore et al. (2016) have used the photonic microscope to observe hygroscopic growth properties of big-size particles. Our method and the size of particle are different from previous studies, but we obtained a consistent DRH to those in Tang et al. (1982), Cruz and Pandis (2000), and Estillore et al. (2016). Since the particle size ( 100 nm) in this study is much smaller than those in previous studies and is not influenced by Kelvin effect, we can capture the hygroscopic growth properties of nanoparticles on real time with the FTIR spectroscopy.

The AS nanoparticle continues to be humidified after deliquescence, resulting in a further increase in $\mathrm{OH}$ area due to continuous water uptake. However, the peak position 
of the sulfate keeps constant regardless of $\mathrm{RH}$, indicating that the AS is still in the aqueous phase after deliquescence. As the increase in $\mathrm{RH}$, the nanoparticle volume increases but its mass keeps constant, resulting in a decrease in concentration and peak area. Figure S1 compares the predicted $M_{\text {water }} / M_{0}$ ( $M_{\text {water }}$ is the mass of liquid water in the nanoparticles; $M_{0}$ is the initial mass of nanoparticles) ratio from E-AIM (UNIFAC model) (http://www.aim.env.uea.ac.uk/aim/aim.php) and the measured hygroscopic properties (Measured $M_{\text {water }}$ and $M_{0}$ are calculated from the IR spectroscopy in Sect. 2.1) from the FTIR spectra for the AS particles with a dry diameter of $100 \mathrm{~nm}$. The results show that the predicted and measured $M_{\text {water }} / M_{0}$ results are consistent after deliquescence, indicating continuous particle water uptake with the increase in RH. It indicated FTIR could be a method to quantitative analysis $M_{\text {water }}$ and $M_{0}$.

Figure 4 shows the FTIR spectral characteristics of the OA nanoparticles under humidity conditions from $40 \%$ to $90 \%$ (RH). The results for the OA nanoparticle differ from those for AS. Throughout the humidification processes, the $\mathrm{O}-\mathrm{H}$ stretching peak at $3250 \mathrm{~cm}^{-1}$ for liquid water was not detected, indicating that liquid water is not absorbed by the $\mathrm{OA}$. The peaks at $3400 \mathrm{~cm}^{-1}$ and $1240 \mathrm{~cm}^{-1}$ are for $\mathrm{O}-\mathrm{H}$ and the $\mathrm{C}=\mathrm{O}$ stretching vibration of OA (Jing et al., 2016), respectively. It shows that the positions and areas of these peaks are also independent of $\mathrm{RH}$. This verifies that no water is absorbed and that no deliquescence transition occurs within the whole RH range.

Figure 5 compares the predicted and measured $M_{\text {water }} / M_{0}$ results for the AS/OA and $\mathrm{AS} / \mathrm{AN}$ nanoparticle mixtures during the humidification processes from $60 \%$ to $95 \%$ (RH). The predicted and measured $M_{\text {water }} / M_{0}$ values showed similar behaviour during these humidification processes. This means that the liquid water absorbed by pure and mixed particles could be measured on real time using the FTIR spectroscopy.

\subsection{Hygroscopic growth of pure and mixed-component nanoparticles}

With the results derived from the FTIR measurements, we calculated the GFs for both pure and mixed nanoparticles via equation (4) and investigated their variabilities with respect to the changes in RH. Figure 6 compares the measured and predicted GF for both pure and mixed nanoparticles under the humidity conditions from $50 \%$ to $95 \%$. The measured and predicted GF are in good agreement. The GF can be obtained precisely using the H-TDMA technique via a direct measurement to the aerosol diameter. In this study, the GFs for both pure and mixed compounds are calculated with liquid water content and the relative masses of dry compound obtained from FTIR 

components in this study are in good agreement with those from previous studies(Jing et al., 2016; Braban et al,. 2003).

The GF curves can be used to investigate the sensitivity of particle volume to RH. If the $\mathrm{RH}$ is less than $80 \%$, the AS nanoparticles are in a stable crystal state that is immune to water and the size of these particles keeps constant. At the RH of $79.9 \pm$ $0.10 \%$, deliquescence occurs, the nanoparticle volume grows up sharply by up to approximately 3.1 times and transforms from the crystalline to aqueous phase. As RH keeps increasing after deliquescence, the AS nanoparticles become fully liquid droplets, and their volumes keep increase due to further water uptake.

Since both the AS/AN and AS/OA mixed nanoparticles absorb liquid water below their DRH, their GF curves differ from the pure AS particle, and their DRH values were lower than that of the AS. The results are in good agreement with previous studies(Seinfeld and Pandis, 2016) which measure GF by H-TDMA. As a result, FTIR measurement technique in this study provide a real-time method to characterize the hygroscopic growth of aerosols.

\subsection{Phase transition dynamics of pure AS nanoparticles}

Although FTIR measurements can be used to characterize the liquid water content and functional groups contained in the nanoparticles during the humidification process, it is difficult to separate the absorption peaks of the nanoparticles (especially for organic compounds) since these absorption peaks are overlapped. In contrast, 2D-IR spectroscopic technique can resolve the overlapping peaks(McKelvy et al., 1998; Du et al., 2021) and, more importantly, can provide detailed information about the dynamic deliquescence processes of the functional groups(Noda and Ozaki, 2014). Synchronous correlation maps reflect the simultaneous changes that occur in the two separate spectral intensity variations. Asynchronous spectra can be used to identify the occurrence sequential order of the hydration interactions caused by external perturbations (Jing et al. 2016).

Figure 7 shows the synchronous and asynchronous correlation maps derived from the 2D-IR measurements for the pure AS compounds. The corresponding correlation maps are not shown for the OA nanoparticles because they absorbed no water and thus presented no deliquescence transition during the humidification process. From the 
synchronous map, one main red/positive $(3250,1097)$ auto-peak was observed for the AS nanoparticles in the 50-90\% RH range, which indicated the existence of coherent variation in those spectral intensities. In addition, the existence of coupling of the spectral intensity variations for the $\mathrm{O}-\mathrm{H}$ stretching peak for liquid water and the symmetrical stretching vibration for the sulfate in the aqueous AS is also possible. Furthermore, two main blue/negative $(1112,1097)$ and $(3250,1112)$ auto-peaks were also observed for the AS nanoparticles, which indicates that the $\mathrm{O}-\mathrm{H}$ stretching peak intensity is increasing, while the symmetrical stretching vibration for the sulfate when hydrated with $\mathrm{NH}_{4}{ }^{+}$in the solid is decreasing. The blue/negative $(1112,1097)$ autopeak indicates that the intensity of the symmetrical stretching vibration for the sulfate in the aqueous AS increased, while that in the solid AS decreased, and these two chemical bonds can be converted into each other. This behavior can thus be explained by the fact that $\mathrm{NH}_{4}{ }^{+}$particles hydrated with $\mathrm{SO}_{4}{ }^{2-}$ are being replaced by $\mathrm{H}_{2} \mathrm{O}$ molecules with increase in $\mathrm{RH}$.

In addition to the synchronous map, the asynchronous map indicates the sequential changes in the spectral intensities in response to the hygroscopic activities. Two main red/positive $(3250,1097)$ and $(1112,1097)$ and two main blue/negative $(3250,1112)$ and $(1097,1112)$ auto-peaks are observed for the AS nanoparticles, which indicated that the peaks changed in the order from $(1112) \mathrm{cm}^{-1}>(3250) \mathrm{cm}^{-1}>(1097) \mathrm{cm}^{-1}$. The intensity of the symmetrical stretching vibration for the sulfate when hydrated with $\mathrm{NH}_{4}{ }^{+}$in the solid would decrease, and then water molecules would attach to the surface of the solid $\left(\mathrm{NH}_{4}\right)_{2} \mathrm{SO}_{4}$ (Yeşilbaş and Boily, 2016); finally, $\mathrm{NH}_{4}{ }^{+}$particles hydrated with $\mathrm{SO}_{4}{ }^{2-}$ are replaced by the $\mathrm{H}_{2} \mathrm{O}$ molecules and the AS nanoparticles then become fully liquid droplets. This indicates that the surface-limited processes may control the water transport to the AS.

\subsection{Phase transition dynamics of mixed nanoparticles}

Figure 8 shows the synchronous and asynchronous correlation maps for the AS/AN mixture nanoparticles. In the synchronous map, one main red/positive $(3250,1097)$ and two blue/negative $(1320,1112)$ and $(3250,1112)$ auto-peaks were observed for the AS/AN nanoparticles (mass ratio of 1:1) in the RH range from 50-90\%. This can be explained by the fact that $\mathrm{NH}_{4}{ }^{+}$particles hydrated with $\mathrm{SO}_{4}{ }^{2-}$ and $\mathrm{NO}_{3}{ }^{-}$are replaced by the $\mathrm{H}_{2} \mathrm{O}$ molecules with increasing $\mathrm{RH}$. In the asynchronous map, three main red/positive $(3250,1097),(1097,1112)$, and $(1320,1097)$ and two main blue/negative 
$315(3250,1320)$ and $(1112,1097)$ auto-peaks are observed, which indicates that the peaks change in the order from (1320) $\mathrm{cm}^{-1}>(3250) \mathrm{cm}^{-1}>(1097) \mathrm{cm}^{-1}>(1112) \mathrm{cm}^{-1}$. This indicates that the nitrate would be in the aqueous solution at a lower RH than the sulfate because the AN has a lower DRH (RH=74.3 $\pm 0.4 \%$, (Seinfeld and Pandis, 2016; Tang and Munkelwitz, 1993)), and the characteristics of the nitrate when hydrated with water differ from those of the sulfate. Furthermore, the hydrolysis reaction mechanism for the sulfate in AS/AN may differ from that for the pure sulfate. One possible explanation for this phenomenon is that the nitrate would begin to absorb water at low $\mathrm{RH}$, which enhances the dissolution of the AS. Therefore, the $\mathrm{NH}_{4}{ }^{+}$particles that would be hydrated with the sulfate are replaced by the $\mathrm{H}_{2} \mathrm{O}$ molecules, and the intensity of the symmetrical stretching vibration for the sulfate in the solid AS nanoparticles would then decrease (Jing et al., 2016).

Figure 9 shows the synchronous and asynchronous correlation maps for the AS/OA mixture nanoparticles. In the synchronous map, one main red/positive (3260, $1080)$ and two main blue/negative $(1112,1050)$ and $(3250,1112)$ auto-peaks were observed for the AS/OA nanoparticles in the RH range from 50-90\%. The two blue/negative auto-peaks are transformed into each other, and the intensity of the symmetrical stretching vibration for the sulfate in aqueous form increases while that in the solid form decreases. In the asynchronous map, one main red/positive $(3250,1080)$ and one main blue/negative $(3250,1112)$ auto-peaks are observed, which indicated that the peaks change in the order from $(1112) \mathrm{cm}^{-1}>(3250) \mathrm{cm}^{-1}>(1080) \mathrm{cm}^{-1}$. Therefore, the hydrolysis reaction mechanism for the sulfate in AS/OA may be similar to that for the pure sulfate. The 2D-IR measurements could thus provide a real time method to characterize the dynamic variability of the nanoparticles during the hygroscopic growth process.

\section{Conclusions}

In this work, we demonstrate use of FTIR spectroscopy to measure the hygroscopic growth properties of assembled nanoparticles and combine this technique with 2D-IR spectroscopy to identify the occurrence sequential order of the hydration interactions and provide detailed information about the dynamic deliquescence processes of the functional groups. This approach enabled measurement of the water content and the dry nanoparticle mass to characterize the hygroscopic GF and also further investigation of the deliquescence process, with results that were matched well with those obtained from 
the E-AIM and the H-TDMA technique in the 50-95\% RH range. Furthermore, we used 2D-IR spectroscopic technique to resolve the overlapping peaks and provide detailed information about the dynamic deliquescence processes of the functional groups. For pure AS, the intensity of the symmetrical stretching vibration for the sulfate when hydrated with $\mathrm{NH}_{4}{ }^{+}$in the solid would decrease, and water molecules would then attach to the surface of solid AS before the $\mathrm{NH}_{4}{ }^{+}$particles hydrated with $\mathrm{SO}_{4}{ }^{2-}$ are finally replaced by the $\mathrm{H}_{2} \mathrm{O}$ molecules and the $\mathrm{AS}$ nanoparticles become fully liquid droplets.

For the AS/AN and AS/OA mixtures, both the AN and OA compounds could lower the deliquescence point for AS. However, AN may have changed the hydrolysis reaction mechanism for the sulfate in AS/AN while the OA did not, which resulted in differences between the results obtained for the sulfates in the pure and mixed nanoparticles.

Therefore, 2D-IR spectroscopic technique represents a suitable method for study of the hygroscopic growth micro-dynamics of nanoparticles and would provide insight into the intermolecular interactions that govern the physicochemical properties of the aerosol and enable better understanding of the nanoparticle-water interactions during the phase transitions.

\section{Author contribution}

XW designed the experiment and wrote the paper with contributions from all coauthors; HG contribute to science discussions and suggested analyses; HD and JZ prepared for the humidification system; YC, JW, YY and JL contributed to this work by providing constructive comments; YS contributed to this work by providing constructive comments, review, and editing.

\section{Competing interests}

The authors declare that they have no conflict of interest that could have appeared to influence the work reported in this paper.

\section{Acknowledgments}

This work was supported by the National Natural Science Foundation of China (No. 41905028, 91544218), the Natural Science Foundation of Anhui (No. 2108085MD139), the Science and Technological Fund of Anhui Province for Outstanding Youth (No.1808085J19). We are also grateful to the China Scholarship Council for their 

about the FTIR data processing.

\section{References}

Abbott T. H. \& Cronin T. W.: Aerosol invigoration of atmospheric convection through increases in humidity, Science, 371, 83-85, doi: 10.1126/science.abc5181,2021

Braban C. F., Carroll M. F., Styler S. A. \& Abbatt J. P. D., Phase Transitions of Malonic and Oxalic Acid Aerosols, The Journal of Physical Chemistry A, 107, 6594-6602, doi: https://doi.org/10.1021/jp034483f, 2003

Carlton A. G., Christiansen A. E., Flesch M. M., Hennigan C. J. \& N. Sareen, Multiphase Atmospheric Chemistry in Liquid Water: Impacts and Controllability of Organic Aerosol. Accounts of Chemical Research, DOI: https://doi.org/10.1021/acs.accounts.0c00301, 53, 1715-1723, 2020.

Cruz C. N. \& Pandis S. N., Deliquescence and Hygroscopic Growth of Mixed Inorganic-Organic Atmospheric Aerosol. Environmental Science \& Technology, 34, 4313-4319, doi: 10.1021/es9907109, 2000

Dong J.-L., Li X.-H., Zhao L.-J., Xiao H.-S., Wang F., Guo X. \& Zhang Y.-H., Raman Observation of the Interactions between NH4+, SO42-, and H2O in Supersaturated (NH4)2SO4 Droplets. The Journal of Physical Chemistry B, 111, 12170-12176, doi:10.1021/jp072772o, 2007.

Downing H. D. \& Williams D., Optical constants of water in the infrared. Journal of Geophysical Research, 80, 1656-1661, https://doi.org/10.1029/JC080i012p01656, 1975

Du H., G. Guo Yu, M. \& Xu H., Investigation of carbon dynamics in rhizosphere by synchrotron radiation-based Fourier transform infrared combined with two dimensional correlation spectroscopy. Science of The Total Environment, 762, 143078, https://doi.org/10.1016/j.scitotenv.2020.143078, 2021

Esat K., David, G. Poulkas T., Shein M. \& Signorell R., Phase transition dynamics of single optically trapped aqueous potassium carbonate particles. Phys Chem Chem Phys, 20, 11598-11607, https://doi.org/10.1039/C8CP00599K, 2018

Estillore A. D., Hettiyadura A. P. S., Qin Z., Leckrone E., Wombacher B., Humphry T., Stone E. A.\& Grassian V. H.,Water Uptake and Hygroscopic Growth of Organosulfate Aerosol. Environmental Science \& Technology, 50, 4259-4268, https://doi.org/10.1021/acs.est.5b05014, 2016

Fan J., Rosenfeld D., Zhang Y., Giangrande S. E., Li Z.,. Machado L. A. T, Martin S. T., Yang Y., Wang J., Artaxo P., Barbosa H. M. J., Braga R. C., Comstock J. M., Feng Z., Gao W., Gomes H. B., Mei F., Pöhlker C., Pöhlker M. L., Pöschl U. \& de Souza R. A. F., Substantial convection and precipitation enhancements by ultrafine aerosol particles. Science, 359, 411, doi: 10.1126/science.aan8461, 2018

Gopalakrishnan Jungwirth S., P., Tobias D. J. \&. Allen H. C, Air-Liquid Interfaces of Aqueous Solutions Containing Ammonium and Sulfate: Spectroscopic and Molecular Dynamics Studies. The Journal of Physical Chemistry B, 109, 8861-8872, https://doi.org/10.1021/jp0500236, 2005.

Gysel M., Weingartner E. \& Baltensperger U., Hygroscopicity of Aerosol Particles at Low Temperatures. 2. Theoretical and Experimental Hygroscopic Properties of Laboratory Generated Aerosols. Environmental Science \& Technology, 36, 63-68, doi:10.1021/es010055g, 2002 
organic aerosols and their internal mixtures with ammonium sulfate. Atmos. Chem. Phys., 16, 4101-4118, https://doi.org/10.5194/acp-16-4101-2016, 2016.

Kirkby J., Curtius J., Almeida J., Dunne E., Duplissy J., Ehrhart S., Franchin A., GagnéS., Ickes L., Kürten A., Kupc A., Metzger A., Riccobono F., Rondo L., Schobesberger S., Tsagkogeorgas G., Wimmer D., Amorim A., Bianchi F., Breitenlechner M., David A., Dommen J., Downard A., Ehn M., Flagan R. C., Haider S., Hansel A., Hauser D., Jud W., Junninen H., Kreissl F., Kvashin A., Laaksonen A., Lehtipalo K., Lima J., Lovejoy E. R., Makhmutov V., Mathot S., Mikkilä J., Minginette P., Mogo S., Nieminen T., Onnela A., Pereira P., Petäjä T., Schnitzhofe r R., Seinfeld J. H., Sipilä M., Stozhkov Y., Stratmann F., ToméA., Vanhanen J., Viisanen Y., Vrtala A., Wagner P. E., Walther H., Weingartner E., Wex H., Winkler P. M., Carslaw K. S., Worsnop D. R., Baltensperger U. \& Kulmala M., Role of sulphuric acid, ammonia and galactic cosmic rays in atmospheric aerosol nucleation. Nature, 476, 429-433, https://www.nature.com/articles/nature10343, 2011.

Kuai Y., Xie Z., Chen J., Gui H., Xu L., Kuang C., Wang P., Liu X., Liu J., Lakowicz J. R. \& Zhang D., Real-Time Measurement of the Hygroscopic Growth Dynamics of Single Aerosol Nanoparticles with Bloch Surface Wave Microscopy. ACS Nano, 14, 9136-9144, https://doi.org/10.1021/acsnano.0c04513, 2020

Lee S.-H. \& Allen H. C., Analytical Measurements of Atmospheric Urban Aerosol. Analytical Chemistry, 84, 1196-1201, https://doi.org/10.1021/ac201338x, 2012.

Martin S. T., Phase Transitions of Aqueous Atmospheric Particles. Chemical Reviews, 100, 3403-3454, doi: 10.1021/cr990034t, 2000.

Matsumura T. \& Hayashi M., Hygroscopic Growth of an (NH 4 ) 2 SO 4 Aqueous Solution Droplet Measured Using an Environmental Scanning Electron Microscope (ESEM). Aerosol Science and Technology, 41, 770-774, doi: 10.1080/02786820701436831, 2007.

McKelvy M. L., Britt T. R., Davis B. L., Gillie J. K., Graves F. B.\& Lentz L. A., Infrared Spectroscopy. Analytical Chemistry, 70, 119-178, doi:10.1021/a1000006w, 1998.

Nájera J. J. \& Horn A. B., Infrared spectroscopic study of the effect of oleic acid on the deliquescence behaviour of ammonium sulfate aerosol particles. Physical Chemistry Chemical Physics, 11, 483-494, https://doi.org/10.1039/B812182F,2009.

Nguyen T. K. V., Zhang Q., Jimenez J. L., Pike M. \& Carlton A. G., Liquid Water: Ubiquitous Contributor to Aerosol Mass. Environmental Science \& Technology Letters, 3, 257-263, https://doi.org/10.1021/acs.estlett.6b00167, 2016.

Noda I. \& Ozaki Y.. Two-Dimensional Correlation Spectroscopy - Applications in Vibrational and Optical Spectroscopy. 15-38. John Wiley \& Sons, 2014.

Richard D. S., Trobaugh K. L., Hajek-Herrera J., Price C. L., Sheldon C. S., Davies J. F. \& Davis R. D., Ion-molecule interactions enable unexpected phase transitions in organic-inorganic aerosol. Science Advances, 6, eabb5643, doi: 10.1126/sciadv.abb5643, 2020.

Riemer N., Ault A. P., West M., Craig R. L.\& Curtis J. H., Aerosol Mixing State: Measurements, Modeling, and Impacts. 57, 187-249, https://doi.org/10.1029/2018RG000615, 2019.

Ruehl C. R., Davies J. F. \& Wilson K. R. J. S., An interfacial mechanism for cloud droplet formation on organic aerosols. 351, 1447-1450, doi:10.1126/science.aad4889, 2016.

Steinfeld J. I. \& Pandis S. N.. Atmospheric Chemistry and Physics: From Air Pollution to Climate Change,3rd ed. John wiley \& Sons, 2016.

Tang I. N. The relative importance of atmospheric sulfates and nitrates in visibility reduction. 

1982.

Tang I. N. \& Munkelwitz H. R., Aerosol growth studies-III ammonium bisulfate aerosols in a moist atmosphere. Journal of Aerosol Science, 8, 321-330, doi:10.1016/0021-8502(77)90019-2, 1977.

Tang I. N. \& Munkelwitz H. R., Composition and temperature dependence of the deliquescence properties of hygroscopic aerosols. Atmospheric Environment. Part A. General Topics, 27, 467473, https://doi.org/10.1016/0960-1686(93)90204-C, 1993.

Tang I. N. \& Munkelwitz H. R., Water activities, densities, and refractive indices of aqueous sulfates and sodium nitrate droplets of atmospheric importance. Journal of Geophysical Research: Atmospheres, 99, 18801-18808, https://doi.org/10.1029/94JD01345, 1995.

Tang M., Cziczo D. J.\& Grassian V. H. Interactions of Water with Mineral Dust Aerosol: Water Adsorption, Hygroscopicity, Cloud Condensation, and Ice Nucleation. Chemical Reviews, 116, 4205-4259, https://doi.org/10.1021/acs.chemrev.5b00529, 2016.

Vogel A. L., Schneider J., Müller-Tautges C., Phillips G. J., Pöhlker M. L., Rose D., Zuth C., Makkonen U., Hakola H., Crowley J. N., Andreae M. O., Pöschl U.\& Hoffmann T., Aerosol Chemistry Resolved by Mass Spectrometry: Linking Field Measurements of Cloud Condensation Nuclei Activity to Organic Aerosol Composition. Environmental Science \& Technology, 50, 1082310832, https://doi.org/10.1021/acs.est.6b01675, 2016.

Wang N., Jing B., Wang P., Wang Z., Li J., Pang S., Zhang Y. \& Ge M., Hygroscopicity and Compositional Evolution of Atmospheric Aerosols Containing Water-Soluble Carboxylic Acid Salts and Ammonium Sulfate: Influence of Ammonium Depletion. Environmental Science \& Technology, 53, 6225-6234, https://doi.org/10.1021/acs.est.8b07052, 2019.

Wang X., Jing B., Tan F., Ma J., Zhang Y.\& Ge M., Hygroscopic behavior and chemical composition evolution of internally mixed aerosols composed of oxalic acid and ammonium sulfate. Atmos. Chem. Phys., 17, 12797-12812, https://doi.org/10.5194/acp-17-12797-2017, 2017.

Wei X., Zhang J., Schwab J., Gao M., Gui H.\& Liu J., Aerosol Pollution Characterization before Chinese New Year in Zhengzhou in 2014. Aerosol and Air Quality Research, 19, 1294-1306, doi: 10.4209/aaqr.2018.06.0226, 2019.

Xu W., Kuang Y., Bian Y., Liu L., Li F., Wang Y., Xue B., Luo B., Huang S., Yuan B., Zhao P. \& Shao M., Current Challenges in Visibility Improvement in Southern China. Environmental Science \& Technology Letters, 7, 395-401, https://doi.org/10.1021/acs.estlett.0c00274, 2020.

Yeşilbaş M. \& Boily J.-F., Particle Size Controls on Water Adsorption and Condensation Regimes at Mineral Surfaces. Scientific Reports, 6, 32136, doi: 10.1038/srep32136, 2016. 
Table

Table 1. Properties of chemicals used in this study

\begin{tabular}{|c|c|c|c|}
\hline Chemical compound & Mol wt, g/mol & Density, $\mathrm{g} / \mathrm{cm}^{3}$ & $\begin{array}{c}\text { Solubility, } \\
\text { g per } 100 \mathrm{~cm}^{3} \mathrm{H}_{2} \mathrm{O}, 20^{\circ} \mathrm{C}\end{array}$ \\
\hline$\left(\mathrm{NH}_{4}\right)_{2} \mathrm{SO}_{4}(\mathrm{AS})$ & 132.14 & 1.769 & 75.4 \\
\hline $\mathrm{NaNO}_{3}(\mathrm{AN})$ & 84.99 & 2.257 & 88 \\
\hline Oxalic acid (OA) & 90.04 & 1.900 & 9.52 \\
\hline
\end{tabular}

\section{Figures}
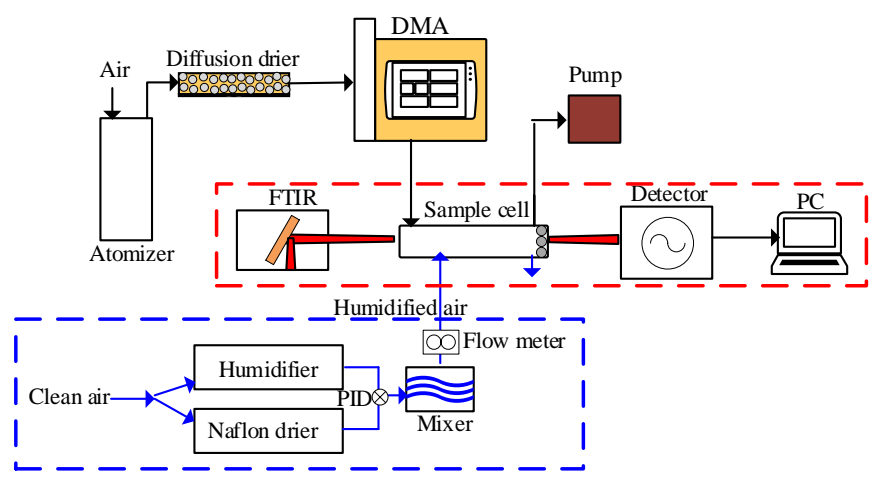

Figure 1. Diagrammatic sketches of the experimental system used to measure nanoparticle hygroscopicity. 


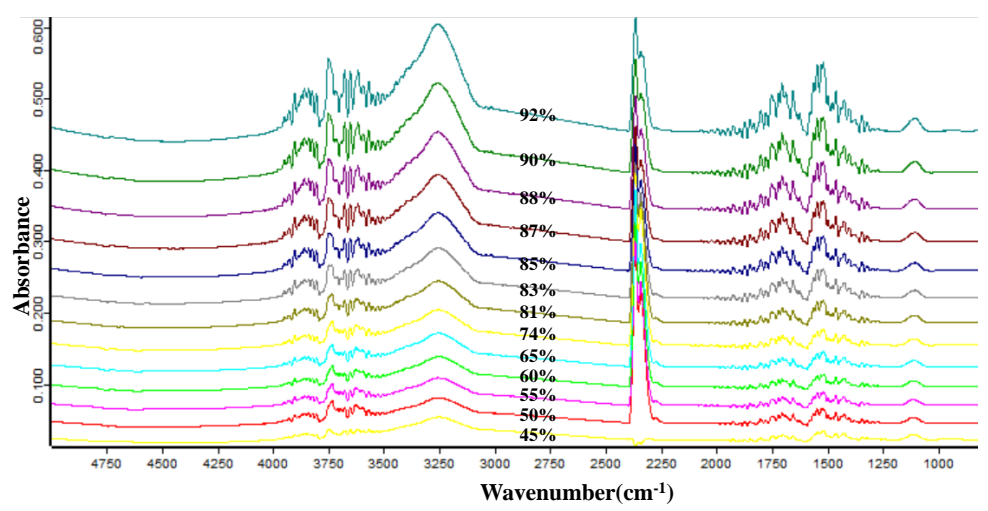

Figure 2. FTIR spectral characteristics of the AS nanoparticles under humidity conditions from $45 \%$ to $92 \%$.

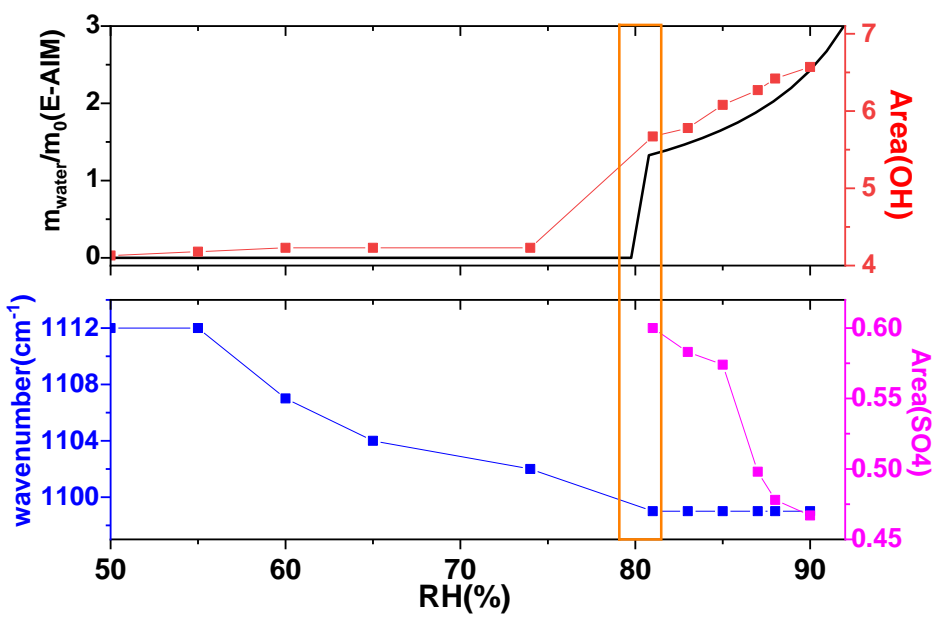

Figure 3. Comparison of the predicted $\mathrm{m}_{\mathrm{water}} / \mathrm{m}_{0}$ and measured hygroscopic properties of AS particles from FTIR with a dry diameter of $100 \mathrm{~nm}$ in the humidification process as a function of RH. The black curves show the E-AIM (UNIFAC) predictions. 


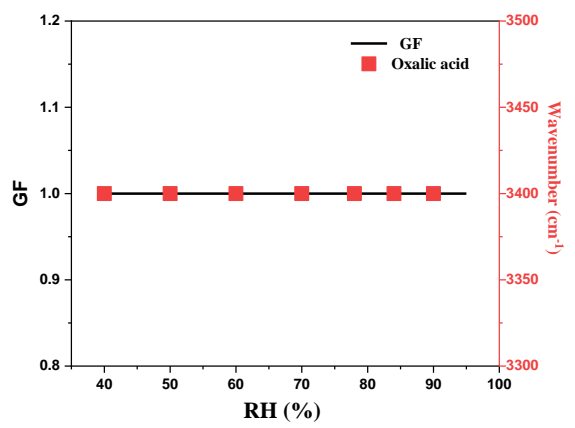

Figure 4. FTIR spectra of OA nanoparticles with a dry diameter of $100 \mathrm{~nm}$.

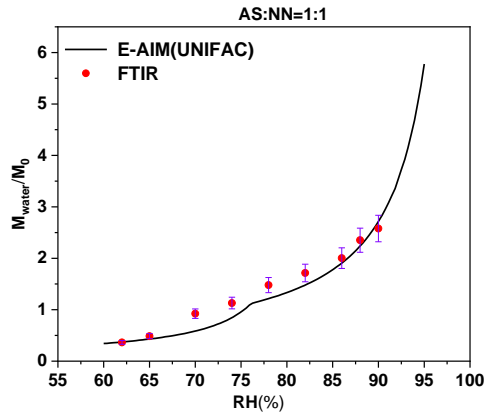

(a) AS/AN

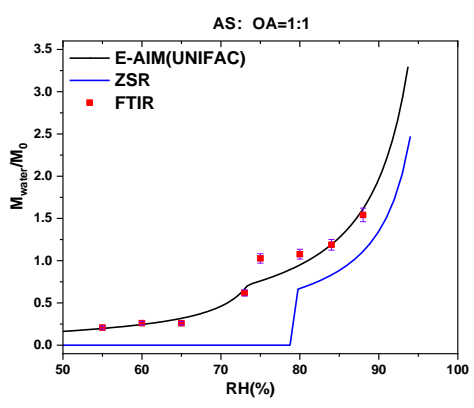

(b) $\mathrm{AS} / \mathrm{OA}$

Figure 5. Comparison of the predicted $M_{\text {water }} / M_{0}$ results and measured hygroscopic properties from the FTIR spectra of (a) AS/OA and (b) AS/AN particles with a dry diameter of $100 \mathrm{~nm}$ during the humidification process as a function of the RH. The black curves represent the E-AIM (UNIFAC) predictions. 

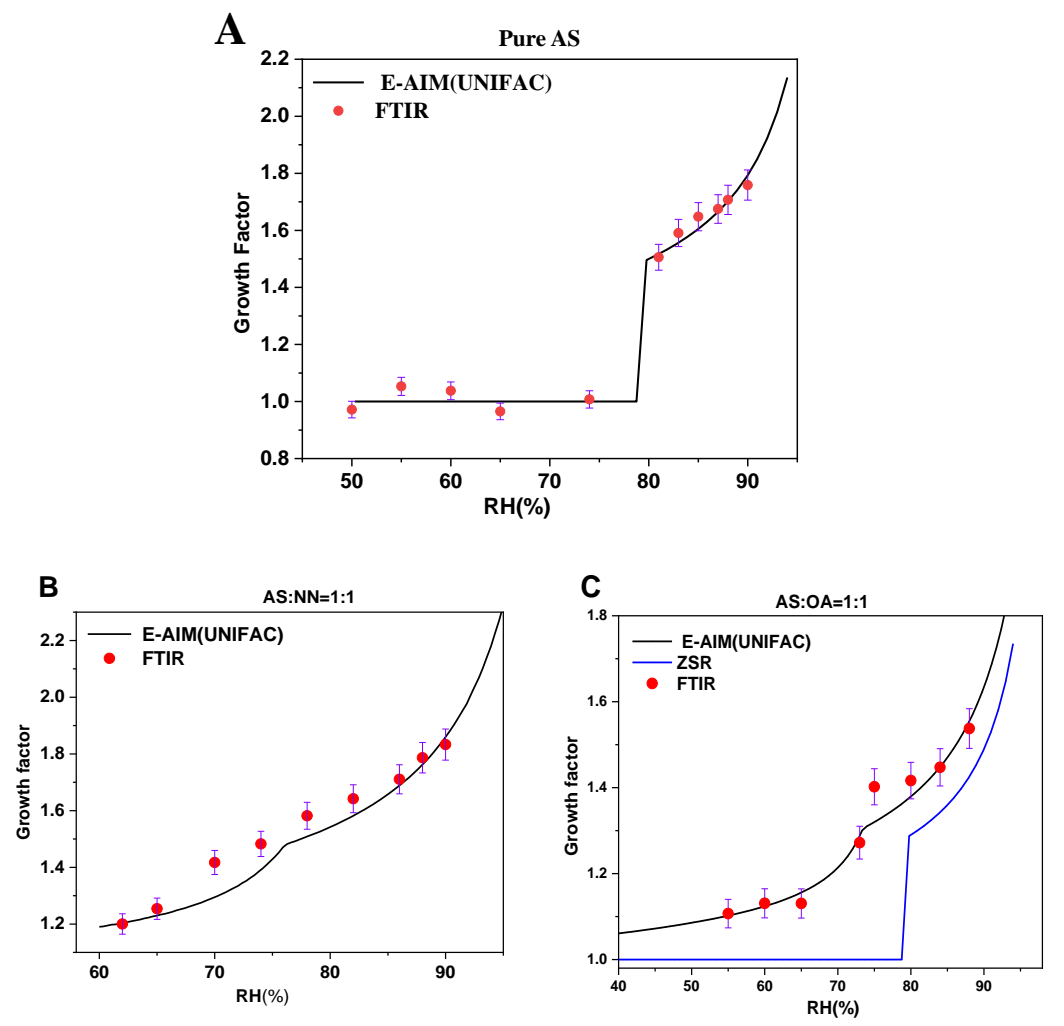

545 Figure 6 Comparison of the measured and predicted growth factor (GF) values of different component nanoparticles: (A) pure AS; (B) AS/AN; and (C) AS/OA. The error bars are shown.

550

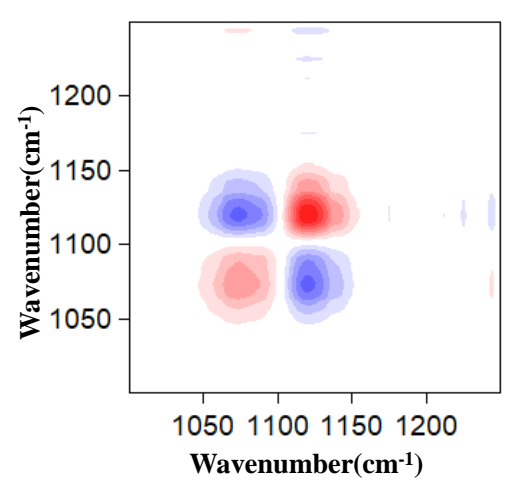

Synchronous

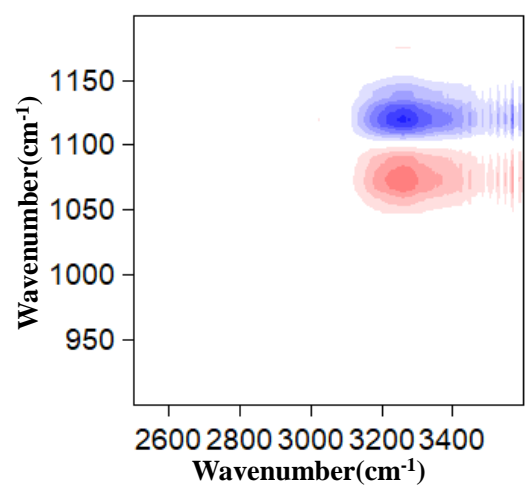

Synchronous 


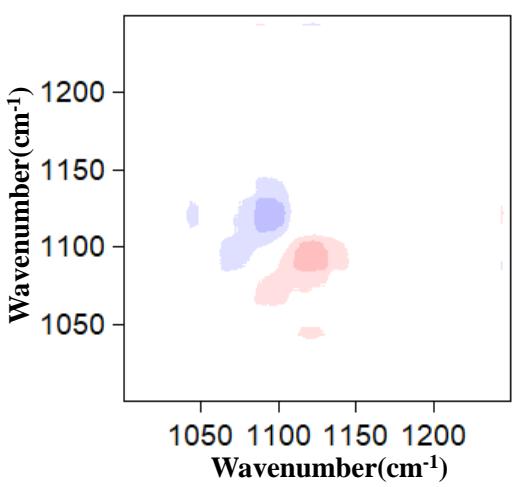

Asynchronous

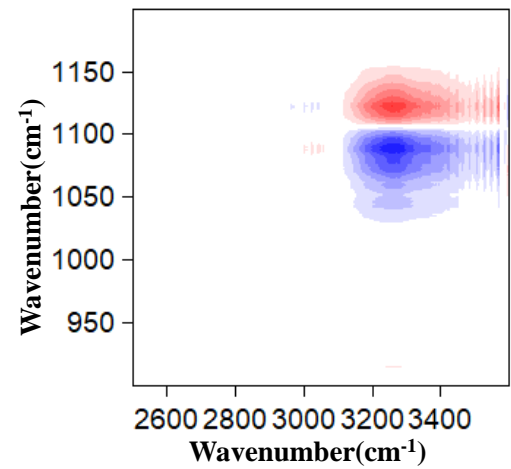

Asynchronous

555 Figure 7 Aynchronous and asynchronous two-dimensional correlation maps generated from the $4000-800 \mathrm{~cm}^{-1}$ region of the FTIR spectra for AS. Red and blue represent the positive and negative correlations, respectively.
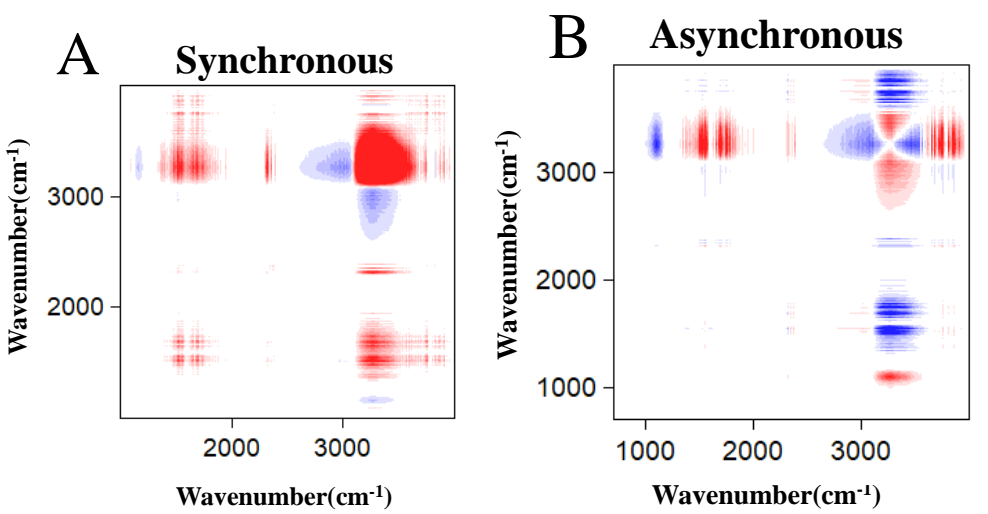

Figure 8. Synchronous and asynchronous two dimensional correlation maps generated in the $800-$ $4000 \mathrm{~cm}^{-1}$ region of the FTIR spectra for AS/AN. Red and blue represent the positive and negative correlations, respectively. 


\section{A Synchronous}
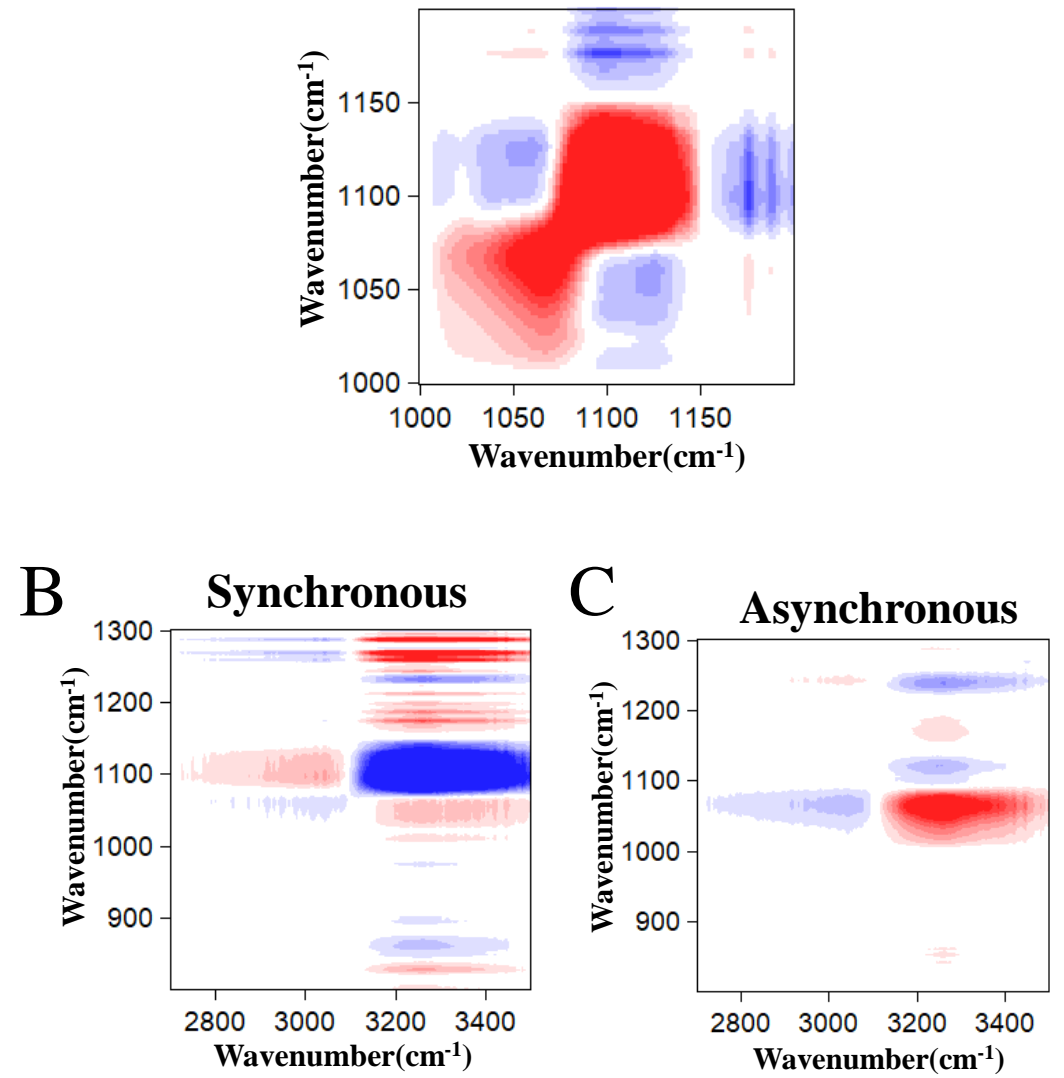

Figure 9. Two dimensional synchronous and asynchronous correlation maps generated in the $800-$ $4000 \mathrm{~cm}^{-1}$ region of the FTIR spectra for AS/OA. Red and blue represent positive and negative correlations, respectively. (A) Synchronous 2D-IR correlation spectrum; (B) synchronous 2D-IR correlation spectrum; (C) asynchronous 2D-IR correlation spectrum; 